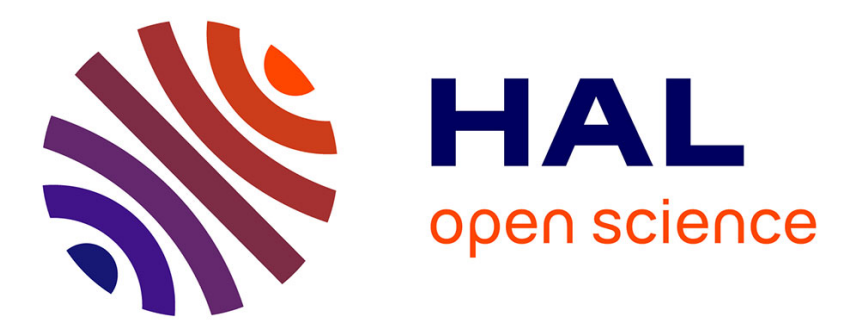

\title{
Radical cation transfer in a guanine pair: an insight to the G-quadruplex structure role using constrained DFT/MM
}

\author{
Ranjitha Ravindranath, Padmabati Mondal, Natacha Gillet
}

\section{To cite this version:}

Ranjitha Ravindranath, Padmabati Mondal, Natacha Gillet. Radical cation transfer in a guanine pair: an insight to the G-quadruplex structure role using constrained DFT/MM. Theoretical Chemistry Accounts: Theory, Computation, and Modeling, 2021, 140 (7), 10.1007/s00214-021-02787-0 . hal03424034

\section{HAL Id: hal-03424034 \\ https://hal.science/hal-03424034}

Submitted on 10 Nov 2021

HAL is a multi-disciplinary open access archive for the deposit and dissemination of scientific research documents, whether they are published or not. The documents may come from teaching and research institutions in France or abroad, or from public or private research centers.
L'archive ouverte pluridisciplinaire HAL, est destinée au dépôt et à la diffusion de documents scientifiques de niveau recherche, publiés ou non, émanant des établissements d'enseignement et de recherche français ou étrangers, des laboratoires publics ou privés. 


\title{
Radical Cation Transfer in a Guanine Pair: an Insight to the G-Quadruplex Structure Role using Constrained DFT/MM
}

\author{
Ranjitha Ravindranath - Padmabati \\ Mondal · Natacha Gillet
}

Received: date / Accepted: date

\begin{abstract}
DNA G-Quadruplex are highly sensitive to oxidation as their structures include $\pi$-stacked guanine quartets allowing fast hole transfer between the nucleobases. These transfers can be described using vertical energy gap and electronic coupling between the different diabatic states at play in a guanine pair. Using classical molecular dynamics simulation and the constrained DFT/MM implementation in deMon2k, we determine these quantity for all the interacting guanine pairs of six G-quadruplex structures including one to four quartets and corresponding to different DNA folding. We then described an uni-directional transfer within a quartet, with high electronic coupling and vertical energy gap values, which contrasts with the hole transfer between $\pi$ stacked guanine, bi-directional and corresponding to smaller charge transfer parameters. The influence of the geometrical parameters on the electronic coupling is explored, while the external or internal position of the guanine may impact its oxidation probability according to the vertical energy gaps.
\end{abstract}

Keywords G-Quadruplex · Guanine Oxidation · Charge Transfer · Constrained DFT · QM/MM

\section{Introduction}

DNA G-quadruplexes consist in a noncanonical folding[1] of guanine-rich DNA sequences. Blocks of four interconnected guanines are stacked and trap metallic

Ranjitha Ravindranath

Indian Institute of Science Education and Research (IISER), Tirupati, 517507 India

Padmabati Mondal

Department of Chemistry and Center for Atomic, Molecular and Optical Sciences and Technologies, Indian Institute of Science Education and Research (IISER) Tirupati, Karakambadi Road, Mangalam, Tirupati-517507, Andhra Pradesh, India.

Natacha Gillet at ENS de Lyon, CNRS UMR 5182, Université Claude Bernard Lyon 1, Laboratoire de Chimie, F-69342, Lyon, France E-mail: natacha.gillet@ens-lyon.fr 
cations which contribute to the G-quadruplex stability. Such structures can be found everywhere along the chromosomes but they are very important in the chromosome extremity, called telomere[2, 3]. Telomeres contain a non-coding DNA sequence and are dedicated to genetic information protection along the cell life and division[4,5]. Other G-quadruplexes are present in oncogenes promoters such as c-Myc[6], or used by different pathogens in their regulatory process[7]. Consequently, G-quadruplexes represent a therapeutic target and numerous ligands have been proposed to regulate gene expression or telomerase activity[8].They are also very sensitive to oxidation by UV-light or oxidative stress: guanine has the lowest ionization potential among the nucleobases and can be easily oxidized to form a radical cation guanine. Once oxidized, the highly reactive guanine can undergo several chemical transformation, such as deprotonation, formation of 8-oxoguanines or cross-links proteins or other molecules $[9,10,11,12,13,14]$.

Experimentally, the oxidation of the G-quadruplex structure can be induced using molecular redox partners or UV radiation absorption[11, 15, 16, 12]. The life time of the radical cation guanine, which competes with the deprotonation of a guanine and the formation of a neutral species, reaches the millisecond timescale while the cation disappears in few microsecond in normal B-DNA.[16] In addition to the guanine-rich sequence, G-quadruplex structures benefits from their folding $[17,18,19,20]$ that allows numerous charge transfer within a quartet or between them. As the deprotonation can occur only on the radical cation guanine, the fast transfer of this latter from one guanine to the other may decrease the probability of a proton transfer.

The tunnelling and incoherent hopping charge transfers are triggered by the vertical energy gap and the electronic coupling between the different redox partners. Indeed, the charge transfer rate for nonadiabatic transfer can be expressed as:

$$
k_{E T}=\frac{2 \pi}{h} H_{D A}^{2} F C
$$

where $H_{D A}$ is the electronic coupling between the diabatic electronic states and FC the Franck-Condon factor[21]. The energy gaps $\Delta \mathrm{E}$ between the different redox states enter in the Franck-Condon factor, and, when all the vibrational frequencies are relatively small, this factor can be expressed as a function of the free enthalpy of reaction $\Delta G^{0}$ and the reorganization energy $\lambda$. In the linear response approximation, these two parameters can be determined directly from the vertical energy gap:

$$
\begin{gathered}
\Delta G^{0}=\frac{1}{2}\left(\Delta E_{f}+\Delta E_{i}\right) \\
\lambda=\frac{1}{2}\left(\Delta E_{f}-\Delta E_{i}\right)
\end{gathered}
$$

A consistent evaluation of the driving force and the reorganization energy in a biological system supposes a conformational sampling of the two considered diabatic states. 
Table 1 Different G-Quadruplex structures

\begin{tabular}{llll}
\hline PDB & Topology & Sequence $\left(5^{\prime} \rightarrow 3^{\prime}\right)$ & Origin \\
\hline 2kf8 [24] & Basket & GGGTTAGGGTTAGGGTTAGGGT & Human telomere \\
143d[25] & Anti-parallel & AGGGTTAGGGTTAGGGTTAGGG & Human telomere \\
1xav[26] & Parallel & TGAGGGTGGGTAGGGTGGGTAA & Proto-oncogene promoter \\
2gku[27] & Hybrid-1 & TTGGGTTAGGGTTAGGGTTAGGGA & Modified human telomere \\
6qjo[28] & Left handed & GGTTGGTGTGGTTGGTGTGGTGGTGGTG & synthetic \\
6gz6[29] & Left handed & GTGGTGGTGGTGTTGTGGTGGTGGTGT & Synthetic \\
\hline
\end{tabular}

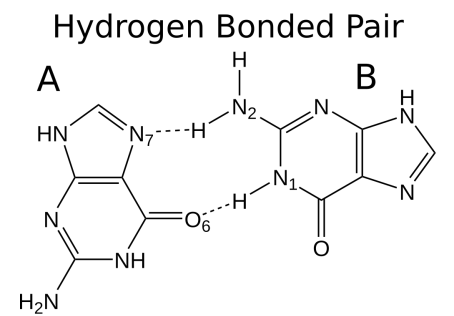

\section{ח-stacked Pair}

A<smiles>CN1CN2CNC(N)C(N)=C1NCN2</smiles>

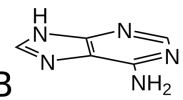
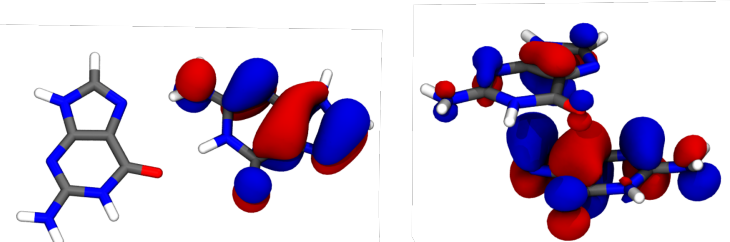

Fig. 1 Chemical representation of the two guanine pairs considered as charge DonorAcceptor couple in gas phase or in $\mathrm{QM} / \mathrm{MM}$ using cDFT. The HOMO orbitals of the neutral pairs in gas phase calculated at PBE0 (50\% Hartree Fock exchange)/Aug-cc-PVTZ level of theory also represented (picture rendered using vmd,[33] isovalue of 0.03 ).

Some computational studies have already explored the influence of the quartets arrangement and stacking in the hole transfer properties using QM or $\mathrm{QM} / \mathrm{MM}$ simulations[22, 23]. While the QM/MM approach focused on a tetrameric parallel-stranded G-quadruplex only,[23] several conformations have been considered in the QM study, [22] with an impact on the transfer, especially on the electronic coupling, but taking into account only two quartets from the G-quadruplex.

In this study, we compare the impact of different G-Quadruplex sequences and conformations (listed in Table 1) using a combination of molecular dynamics simulation (MD) and single point constrained DFT[30, 31] (cDFT)/MM calculation.[32] We define two type of guanine pairs, represented in Figure 1 , one involving two guanines from a quartet linked by hydrogen bonds, the other one consisting in two $\pi$-stacked guanines from two different quartets. We intend to obtain a first evaluation of the influence of the geometry and the environment, including water molecules and nucleobases that are not involved in a quartet, to two charge transfer parameters, namely the diabatic vertical energy gap and the electronic coupling. 


\section{Computational Methods}

\subsection{Molecular Dynamics Simulations}

We selected six G-quadruplex structures that cover different foldings of the DNA strand (see Table 1). All the simulations were performed using Amber 20 package[34] and the Amber parmbsc1[35] force fields for nucleobases. Each Gquadruplex is solvated in TIP3P[36] water rectangular box which dimensions allow a solvent layer of $12 \AA$ around the DNA structure. Sodium cations were added to ensure neutrality. A minimization was run, followed by a heating procedure (heating from 0 to $300 \mathrm{~K}$ in $30 \mathrm{ps}$ with a timestep of $1 \mathrm{fs}$ ). Then, a last equilibration run of 1 ns and a $1 \mu$ s production run were carried out in the NPT ensemble, using the Langevin thermostat and the Berendsen barostat at $300 \mathrm{~K}$ and $1 \mathrm{~atm}$. A timestep of $2 \mathrm{fs}$ was used as covalent bonds involving a hydrogen were constrained using the SHAKE algorithm. Periodic boundary conditions were used, allowing the treatment of the long-range electrostatic interactions with the Particle Mesh Ewald (PME) algorithm[37]. For noncovalent interactions, a cutoff of $12 \AA$ was used.

The trajectories were analysed using the cpptraj tool from amber20 package, [38] first through the hierarchical agglomerative approach for clustering, based on the RMSD of the solute. Some of the representative structures of the obtained cluster are given in Figure S1 in Supporting information. The distance and the twist angle, defined as the dihedral angle involving the center of the quartet and the N9 of each guanine in the considered $\pi$-stacked pair, are then calculated for the representative structure of the selected clusters.

\subsection{Constrained DFT calculations}

All QM and QM/MM calculations were performed using deMon2K[39] and the implementation of CDFT within[40]. PBE0[41] with $50 \%$ of Hartree-Fock exchange was used with the augmented cc-pVTZ basis[42] set and Grimme correction for dispersion[43], as recommended by previous benchmarks.[44] The GEN-A2 auxiliary basis set was employed.[45] The charge constraint was calculated to fit an iterative Hirshfeld atomic charge distribution.[46]

First, a pair of $\pi$-stacked or hydrogen bonded guanines (see Figure 1) was optimized at neutral state to get a gas phase reference. Then, the vertical energy gap and the electronic coupling was calculated for a positively charged system using cDFT. The charge difference was constrained to be equal to one, while one guanine is defined as the electron donor $\mathrm{D}$ and the other as electron acceptor $\mathrm{A}$. The donor-acceptor exchange allows the determination of the diabatic vertical energy gap by subtracting the energy of final $\left(D^{+} A\right)$ and initial $\left(D A^{+}\right)$state and the electronic coupling between the two electronic states (Equation 4).

$$
\Delta E=E_{D^{+} A}-E_{D A^{+}}
$$


The electronic coupling is determined after the orthogonalization of the cDFT $2 \times 2$ diabatic Hamiltonian into the basis spanned by the eigenvectors of an operator reflecting the charge difference between $\mathrm{D}$ and $\mathrm{A}$ as described in ref [47].

Secondly, single point QM/MM calculations were performed on the main structures obtained from cluster analysis of each trajectories using the QM/MM module of deMon2k[32]. For each calculation, the purine ring of two guanines were considered in the QM zone (see Figure 2). A hydrogen link atom was positioned in the N9-C1' bond. The MM area encompasses the atoms in a sphere of $25 \AA$ around the center of the box, and the interactions between the QM and MM systems are treated with an electrostatic embedding. No geometry optimization has been performed. The vertical energy gap and the electronic coupling of the transfer of the radical cation between two $\pi$-stacked or two hydrogen bonded guanines were calculated for each possible pair of the six systems. To increase the size of the QM zone, we added a third purine ring using the same QM/MM boundary treatment in order to get one guanine from each quartet of the $143 \mathrm{~d}$ structure.

\section{Hydrogen Bonded Pair}

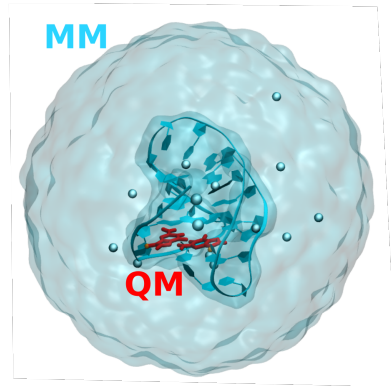

ח-stacked Pair 2 Guanines

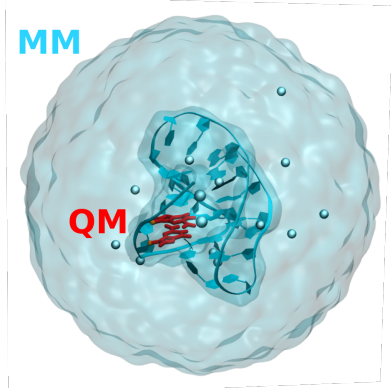

ח-stacked Pair 3 Guanines

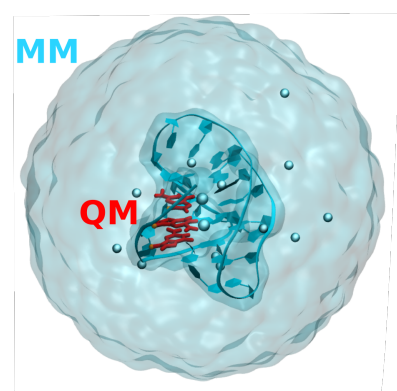

Fig. 2 Representation of the different QM/MM systems for hydrogen bonded pairs and $\pi$-stacked pairs with 2 or $3 \mathrm{QM}$ guanines: QM guanines are in red, link atom in orange and the classical part including nucleobases, cations and water molecule is in cyan.

\section{Results and Discussion}

3.1 Guanine pairs in gas phase

In order to evaluate the impact of the G-quadruplex environment on the charge transfer parameters, we first apply the cDFT approach on a guanine pair in gas phase. Two systems are defined, extracted for a G-quadruplex structure: a pair of $\pi$-stacked guanines and a pair of hydrogen bonded guanines (see Figure 1). A diabatic state is defined by the localization of the positive charge 
Table 2 cDFT diabatic electronic coupling $H_{D A}$ and vertical energy gap $(\Delta \mathrm{E})$ for hydrogen bonded and $\pi$-stacked guanine pairs optimized at the neutral state.

\begin{tabular}{lll}
\hline & $H_{D A}(\mathrm{eV})$ & $\Delta \mathrm{E}(\mathrm{eV})$ \\
\hline H-bond & 0.895 & 0.204 \\
$\pi$-stacked & 0.052 & 0.017 \\
\hline
\end{tabular}

on one guanine fragment, which is ensured by the constraint on the charge localization.

In the hypothesis of a fast charge transfer between the two guanines, the system does not have time to relax and the neutral conformation represent a suitable starting for the diabatic parameters calculation. If the charge transfer occurs in the Marcus theory framework, the optimization of each diabatic state, and even better, some dynamical simulations in each diabatic wells, would be required. However, in guanine-rich DNA, the radical cation moves relatively fast and therefore a neutral conformation can be a consistent starting point to quantify charge transfer parameters.

The diabatic vertical energy gap and electronic coupling between the $\mathrm{G}^{\bullet+} \mathrm{G}$ and $\mathrm{GG}^{\bullet+}$ for both $\pi$-stacked and hydrogen bonded pairs are reported in Table 2. Considering first the hydrogen bonded pair, we obtain a large electronic coupling value and also a relatively high energy gap (but lower than the coupling). In this pair, despite the similar chemical nature of the two partners, the atoms involved in the interactions differ according to the guanine, if it donates its $\mathrm{H} 2$ and $\mathrm{H} 1$ protons or if it accepts them via its $\mathrm{O} 6$ and $\mathrm{N} 7$ atoms. The vertical energy gap indicates that the proton acceptor is the most easily oxidized guanine (guanine A in Figure 1), in agreement with the localization of the HOMO, mostly on the proton donor guanine (B in Figure 1). Furthermore, on the neutral pair, a charge difference of more than 0.43 is observed (see Table $\mathrm{S} 1$ in SI), with a more positive guanine A. When applying the constrained charge difference of 1 , the guanine A can accept the entire positive charge whereas the charge is split between the two guanines when B is supposed to be the electron acceptor (see Table S1 in SI). The hole transfer thus occurs in one way, following the proton donation: from the proton donor guanine to the acceptor one.

On the contrary, the $\pi$-stacked pair presents a very small vertical energy gap, which is consistent with the fact that the geometry is much more symmetric. The neutral HOMO (Figure 1) is delocalized on the two guanines, with a slight predominance on one of them which is not reflected by the atomic charges difference (0.014 see Table S2 in SI). When the charge difference is constrained, each guanine can be fully oxidized with a charge of 1.00 spread out on their respective purine ring. This transfer is thus bi-directional, mostly symmetric, with a relatively high electronic coupling compared to the energy gap. However, the G-quadruplex environment can distort this symmetry and induce a preferential positive charge localization, which will be explored using QM/MM protocol. 
Table 3 Average and standard deviation values of the cDFT diabatic electronic coupling $H_{D A}$ and the absolute value of vertical energy gap $(|\Delta E|)$ for the different hydrogen bonded guanine pairs in a given G-quadruplex structure (cluster 0 only).

\begin{tabular}{lll}
\hline & $H_{D A}(\mathrm{eV})$ & $|\Delta E|(\mathrm{eV})$ \\
\hline 2kf8 & $0.524 \pm 0.300$ & $1.012 \pm 0.619$ \\
143d & $0.671 \pm 0.161$ & $1.063 \pm 0.684$ \\
1xav & $0.648 \pm 0.176$ & $0.916 \pm 0.503$ \\
2gku & $0.659 \pm 0.169$ & $0.966 \pm 0.504$ \\
6qjo & $0.592 \pm 0.251$ & $0.899 \pm 0.394$ \\
6gz6 & $0.586 \pm 0.215$ & $0.900 \pm 0.540$ \\
\hline
\end{tabular}

\subsection{Charge transfer in G-Quadruplex structures}

$1 \mu \mathrm{s}$ classical MD simulations were performed starting from the X-Ray or NMR structures of the different G-quadruplexes we have selected, in order to sample the conformational space of the diverse DNA foldings. None of the trajectories shows large fluctuations from its starting structure. The layout of the different quartet stays similar all along the simulations, while the adjacent nucleobases can adopt different conformations, highlighted by cluster analysis of the trajectories (see Figure S1). Among these conformational fluctuations, some involve a possible $\pi$-stacking of a nucleobase with a quartet which can be otherwise in contact with solvent. Consequently, the close environment of some external guanines can be strongly modified during the molecular dynamics simulation whereas the central quartets undergoes a stable interaction network. As the redox properties are sensitive to the electrostatic field, the impact of these different conformations must be considered.

In the following, we thus report QM/MM calculations on the representative structure of the main cluster of each trajectory and on structures where some guanine close environment is modified. Similarly to gas phase calculation, we consider $\pi$-stacked or hydrogen bonded guanine pairs. First, only the guanines involved in the pair are considered in the QM zone while the other nucleobases and the water are modeled using the classical force field parameters. The electronic couplings and vertical energy gaps, averaged on all pairs of a given G-quadruplex are reported in Tables 3 and 4 (while the value for each pair is given is SI, Tables S3 to S14).

The averaged electronic coupling and vertical energy gap values for hydrogen bonded pairs are similar for all G-quadruplex structures. They also fall in the same range as gas phase values (Table 2), meaning hundreds of meV, with a wide distribution (about half of the average value); however the electronic coupling is lower and the vertical energy gap higher than in gas phase, suggesting that the conformations of guanine pairs within the G-quadruplex structure are less favorable to charge transfer than the optimized structure in gas phase. Within one pair, the hydrogen bonds network triggers the preferential localization of the charge as observed in gas phase: the proton acceptor guanine is always the most likely oxidized guanine with respect to the sign of the vertical 
Table 4 Average and standard deviation values of the cDFT diabatic electronic coupling $H_{D A}$ and the absolute value of vertical energy gap $(|\Delta E|)$ for the different $\pi$-stacked guanine pairs in a given G-quadruplex structure (cluster 0 only).

\begin{tabular}{lll}
\hline & $H_{D A}(\mathrm{eV})$ & $\Delta E \mid(\mathrm{eV})$ \\
\hline 2kf8 & $0.041 \pm 0.030$ & $0.116 \pm 0.138$ \\
143d & $0.060 \pm 0.042$ & $0.189 \pm 0.126$ \\
1xav & $0.075 \pm 0.036$ & $0.304 \pm 0.165$ \\
2gku & $0.091 \pm 0.049$ & $0.295 \pm 0.192$ \\
6qjo & $0.066 \pm 0.045$ & $0.291 \pm 0.232$ \\
6gz6 & $0.094 \pm 0.056$ & $0.371 \pm 0.228$ \\
\hline
\end{tabular}

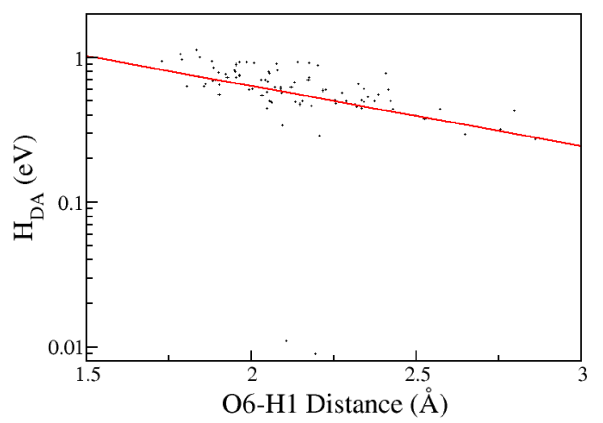

Fig. 3 Electronic coupling $H_{D A}$ between the hydrogen bonded pairs of all G-quadruplexes as a function of O6-H1 distance. Exponential fitting (in red) corresponds to an exponential factor of $-0.957 \pm 0.295 \mathrm{eV}^{-1}$ and a prefactor of $4.297 \mathrm{eV}$.

energy gap. The large value of this latter impedes a backward transfer to the proton donor guanine. Despite this one way transfer, the square arrangement of the quartet allows an oxidation of the four guanines. Then, other parameters can play a role in the tuning of charge transfer. For instance, a decrease of the hydrogen bond distances N7-H2 or O6-H1 leads to an increase of the electronic coupling, as shown in Figures 3 and 4. In agreement with the mono-exponential decay observed for electronic coupling with D-A distance, we fit our data with an exponential curve. We get similar exponential decay factor around -0.95 $\mathrm{eV}^{-1}$ for the two fitting curves but with a relatively large error. Our fitting can suffer from a relative small sampling with two inconsistent points at very low coupling and from the multi-factorial origin of the electronic coupling values. The interactions with the environment and the other quartets can also influence the oxidation preference. The reorganization aspect of the guanines and their environment is not explored by our current calculations, but the wide distribution of the vertical energy gaps underlines the high sensitivity of this transfer to the conformation of the guanine pair and its environment. This can be in agreement to the previously described low transfer rate within a quartet.[22]

The electronic coupling and the diabatic energy gap for the $\pi$-stacked pairs are lower (tens or few hundreds of $\mathrm{meV}$ ) than the hydrogen bond ones (see Ta- 


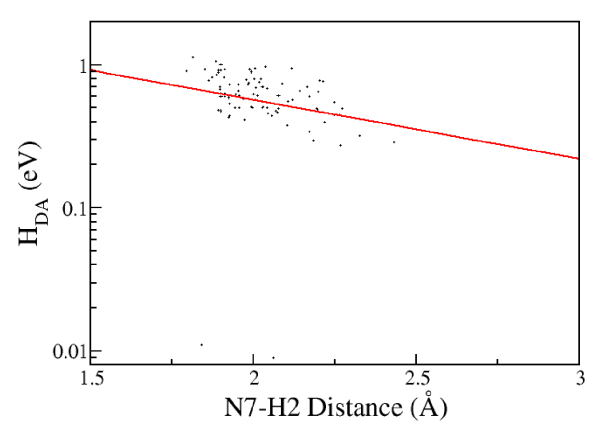

Fig. 4 Electronic coupling $H_{D A}$ between the hydrogen bonded pairs of all G-quadruplexes as a function of N7-H2 distance. Exponential fitting (in red) corresponds to an exponential factor of $-0.953 \pm 0.563 \mathrm{eV}^{-1}$ and a prefactor of $3.822 \mathrm{eV}$.

ble 4), as previously observed in gas phase. They are similar to the previously obtained values in other studies [23]. Considering these values, the transfer is possible in both ways, in agreement with the conductivity of G-quadruplexes or guanine rich DNA sequences.

All the G-quadruplexes structures present similar averaged electronic coupling values despite their different folding. The overlap of the $\pi$ HOMO orbital of the two guanines is crucial for the efficiency of the hole transfer in a $\pi$-stacked pair and tunes the electronic coupling between the two redox states. Consequently, geometrical parameters such as the distance between the purine rings or some angles between them can modulate the electronic coupling value, as shown in the previous study from Lech et al for the twist and shift angles[22]. The Figure 5 represents the electronic coupling scale as a function of guanines distance and twist angle between them (not considering the direction of the rotation or the parallel/anti-parallel relative conformation of the ring). No obvious correlation can be drawn, even if the highest coupling values are obtained for a distance equal or shorter than $4 \AA$ and a twist angle between 15 and 20 degrees. The twist angle distribution of our conformational set is relatively narrow (compare to the one previously published), which can mask the dependence of the electronic coupling on this angle.

Despite their similarities, the averaged vertical energy gaps in Table 4 present more discrepancy than the electronic coupling values. For instance, it is higher for $6 \mathrm{gz} 6$ structure, which contains four quartets, and lower for $2 \mathrm{kf} 8$, where only two full quartets are present (a guanine is missing in the third one). However, this difference cannot be explained by a larger vertical energy gap between the quartet 2 and 3 in 6qjo or 6gz6 structures: considering only these "internal-internal pairs", the absolute vertical energy gap is $0.235 \pm$ $0.161 \mathrm{eV}$, whereas it is $0.285 \pm 0.200$ counting the "internal-external" pairs. This classification must be nevertheless modulated when other nucleobases, which do not belong to a quartet, have a $\pi$-stack interaction with the external quartet. The burying of the guanine also impacts the position of the positive charge when considering the vertical energy gaps with respect to their sign: 


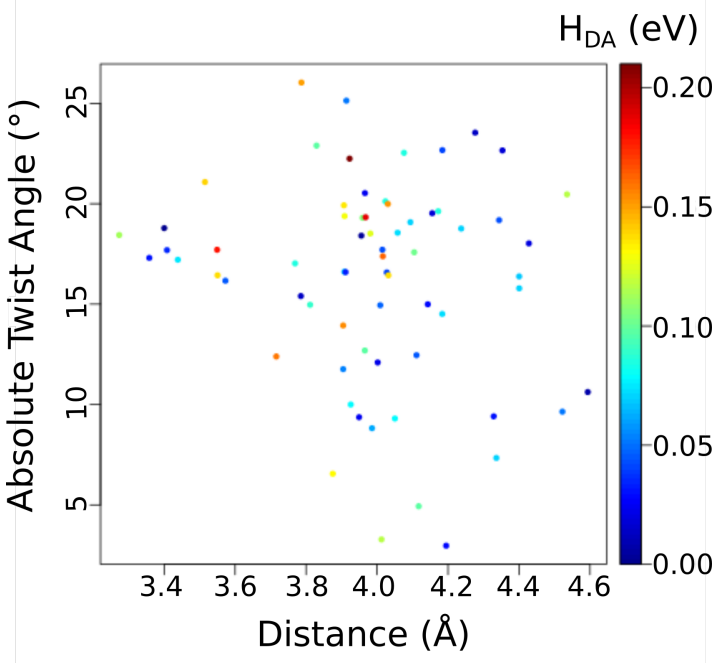

Fig. 5 Electronic coupling $H_{D A}$ between the $\pi$-stacked pairs of all G-quadruplexes as a function of guanine-guanine distance and the twist angle between the two guanines.

most of the time, the state with the positive charge localized on the external guanine is preferred. The internal quartets suffer from the proximity of two cations instead of one whereas the presence of water molecules may help to stabilize the charge more easily than in the G-quadruplex internal quartets. Once again, the presence of a $\pi$-stacked thymine or adenine can tune this preference. The comparison of the different clusters with diverse $\pi$-stacking interactions does not provide a clear definition of their influence: for some pairs, the presence of a guanine or an adenine close to the external guanine decreases the preference for an external localization of the charge (see for example 143d G20G21, or the comparison between cluster 0 and 1 for 1xav G17G18), whereas it seems to play a reversal role for other pairs (A7 for 143d G20G21, A13 for 143d G21G22, A22 for 1xav G10G9, T13 for 2gku G11G10) (see Table S4, S5 and S6 in SI). This divergence may result from a lack of sampling or the vertical energy gap tuning by other parameters, not explored yet. This behavior deserves a more detail study, taking into account the solvent accessibility of each pair and a more dynamical simulations, allowing to sample $\pi$-stacking or water conformations that trigger the stabilization of the positive charges.

Taking together, our results on $\pi$-stacked pairs highlight the importance of the $\pi-\pi$ interactions within and around the redox pair. Because of the quantum nature of such interactions, a classical description of the other nucleobases involved in the $\pi$-stacked chain might be insufficient, but the cost of the QM/MM calculation dramatically increases with the number of atoms in the QM part. We report in Table 5 the comparison between the vertical energy gap for $\pi$ stacked pairs in 143d structure taking into account 2 or 3 guanines. For most 
Table 5 Comparison between the vertical energy gaps from QM/MM calculations containing 2 or 3 guanines of a guanine column in the QM part for $\pi$-stacked pair in $143 \mathrm{~d}$ structure.

\begin{tabular}{llll}
\hline Column & Pair & $|\Delta E|(\mathrm{eV})$ & $|\Delta E|(\mathrm{eV})$ \\
& & 2 Guanines & 3 Guanines \\
\hline \multirow{2}{*}{ G2G3G4 } & G3G2 & 0.201 & 0.143 \\
& G4G3 & -0.047 & -0.099 \\
G8G9G10 & G8G9 & -0.344 & -0.446 \\
& G9G10 & 0.358 & 0.315 \\
G14G15G16 & G15G14 & -0.166 & -0.197 \\
& G16G15 & -0.077 & -0.125 \\
& G20G21 & -0.083 & -0.221 \\
& G21G22 & 0.311 & 0.325 \\
\hline
\end{tabular}

of the pairs, the inclusion of a third guanine in the QM zone increases the vertical energy gap, except for G3G2 and G9G10 pairs. This augmentation ranges between 14 to $138 \mathrm{meV}$ so less than the standard deviation observed for the vertical energy gap distribution. Consequently, increasing the QM zone influences the results but probably in a less important way than the fluctuation of the close environment. However, increasing the size of the QM zone impedes the increasing of the conformational sampling. Consequently, in order to improve the description of the charge transfer in G-quadruplex, one may favor an exhaustive conformational description instead of a better computational description.

Ideally, one should use a polarizable embedding to simulate electron motion as a polarizable environment can have dramatic effect on charge transfer parameters, notably the reorganization energy [48]. Such a QM/MM scheme has been also implemented in deMon2K [32, 49], and can be used instead of a larger QM zone, especially when one wants to simulate the charge transfer itself.

\section{Conclusion}

In this work, we study the influence of the environment of a whole G-quadruplex on the redox properties (diabatic vertical energy gap and electronic coupling) of a pair of guanines, interacting by hydrogen bonds or $\pi-\pi$ interaction, using a cDFT/MM scheme. In gas phase, while the $\pi$-stacked pair is symmetric and no guanine is more easily oxidized, the hydrogen bonded pair presents a directionality of the charge transfer which favors the oxidation of the proton acceptor guanine instead of the proton donor. In a quartet, a symmetry is recovered and even though the directionality of the charge transfer remains, all the guanines can be oxidized thanks to their dual role as proton donor and acceptor. The high electronic coupling, partially tuned by the distances of the hydrogen bonds, should allow a relatively fast charge transfer; however the large discrepancy in the vertical energy gap suggests a strong dependence 
of the transfer to the geometry and the environment. Consequently, the reorganisation energy may represent a limiting factor for this transfer that must be further explored. Besides, the transfer within a quartet competes with a transfer through the $\pi$-stacked pairs. The inter-quartets transfer consists in a bi-directional transfer, with a smaller electronic coupling and vertical energy gap value but fluctuating in a less extended range. The relative position of the quartet and the solvent accessibility may tune the $\pi$ - $\pi$ transfer to modify not only its rate but also the most likely oxidized guanine. Another parameter that must be explored in the future is the nature of the central cation.

Our study provides calculation at DFT level of charge transfers within different oxidized G-quadruplex. It paves the way to a more dynamical study, based on less expensive approaches, parameterized on these first results, to enhance our understanding of the role of the specific folding of G-quadruplex on the long lifetime of radical cation within these structures.

Acknowledgements R.R. is grateful for the IISER-ENS de Lyon fellowship which has financed her visit at the ENS de Lyon. The authors thank the SYSPROD project and AXELERA Pôle de Compétitivité for financial support (PSMN Data Center). This work was performed using HPC resources from GENCI-IDRIS (Grant 2020-A0070800609). N. G. thanks A. de la Lande for providing the deMon2k version with the last cDFT and QM/MM modules and his kind explanations.

\section{Conflict of interest}

The authors declare that they have no conflict of interest.

\section{References}

1. Marchand A, Gabelica V (2016) Folding and misfolding pathways of G-quadruplex DNA. Nucleic Acids Research 44(22):10999-11012, https://academic.oup.com/nar/article-lookup/doi/10.1093/nar/gkw970

2. Moyzis RK, Buckingham JM, Cram LS, Dani M, Deaven LL, Jones MD, Meyne J, Ratliff RL, Wu JR (1988) A highly conserved repetitive dna sequence, (ttaggg)n, present at the telomeres of human chromosomes. Proceedings of the National Academy of Sciences 85(18):6622-6626, https://www.pnas.org/content/85/18/6622, https://www.pnas.org/content/85/18/6622.full.pdf

3. Bryan TM (2020) G-quadruplexes at telomeres: Friend or foe? Molecules 25(16), https://www.mdpi.com/1420-3049/25/16/3686

4. Armanios M, Blackburn EH (2012) The telomere syndromes. Nature Reviews Genetics 13(10):693-704, http://www.nature.com/articles/nrg3246

5. Artandi SE, DePinho RA (2010) Telomeres and telomerase in cancer. Carcinogenesis 31(1):9-18, https://doi.org/10.1093/carcin/bgp268

6. Balasubramanian S, Hurley LH, Neidle S (2011) Targeting G-quadruplexes in gene promoters: a novel anticancer strategy? Nature Reviews Drug Discovery 10(4):261-275, http://www.nature.com/articles/nrd3428 
7. Neidle S (2017) Quadruplex nucleic acids as targets for anticancer therapeutics. Nature Reviews Chemistry 1(5):1-10, http://www.nature.com/articles/s41570-017-0041

8. Harris LM, Merrick CJ (2015) G-quadruplexes in pathogens: A common route to virulence control? PLOS Pathogens 11(2):1-15, https://doi.org/10.1371/journal.ppat.1004562

9. Cadet J, Davies KJA, Medeiros MH, Di Mascio P, Wagner JR (2017) Formation and repair of oxidatively generated damage in cellular DNA. Free Radical Biology and Medicine 107:13-34, http://www.sciencedirect.com/science/article/pii/S0891584916311510

10. Fleming AM, Burrows CJ (2013) G-Quadruplex Folds of the Human Telomere Sequence Alter the Site Reactivity and Reaction Pathway of Guanine Oxidation Compared to Duplex DNA. Chemical Research in Toxicology 26(4):593-607, http://pubs.acs.org/doi/10.1021/tx400028y

11. Balanikas E, Banyasz A, Baldacchino G, Markovitsi D (2019) Populations and Dynamics of Guanine Radicals in DNA strands-Direct versus Indirect Generation. Molecules 24(13):2347, https://www.mdpi.com/1420$3049 / 24 / 13 / 2347$

12. Banyasz A, Balanikas E, Martinez-Fernandez L, Baldacchino G, Douki T, Improta R, Markovitsi D (2019) Radicals Generated in Tetramolecular Guanine Quadruplexes by Photoionization: Spectral and Dynamical Features. The Journal of Physical Chemistry B 123(23):4950-4957, https://pubs.acs.org/doi/10.1021/acs.jpcb.9b02637

13. Zhang X, Jie J, Song D, Su H (2020) Deprotonation of Guanine Radical Cation G $\bullet+$ Mediated by the Protonated Water Cluster. The Journal of Physical Chemistry A 124(29):6076-6083, https://doi.org/10.1021/acs.jpca.0c03748

14. Perrier S, Hau J, Gasparutto D, Cadet J, Favier A, Ravanat JL (2006) Characterization of Lysine-Guanine Cross-Links upon One-Electron Oxidation of a Guanine-Containing Oligonucleotide in the Presence of a Trilysine Peptide. Journal of the American Chemical Society 128(17):57035710, http://pubs.acs.org/doi/abs/10.1021/ja057656i

15. Martinez-Fernandez L, Changenet P, Banyasz A, Gustavsson T, Markovitsi D, Improta R (2019) Comprehensive Study of Guanine Excited State Relaxation and Photoreactivity in G-quadruplexes. J Phys Chem Lett p 5

16. Banyasz A, Martínez-Fernández L, Balty C, Perron M, Douki T, Improta R, Markovitsi D (2017) Absorption of Low-Energy UV Radiation by $\mathrm{Hu}-$ man Telomere G-Quadruplexes Generates Long-Lived Guanine Radical Cations. Journal of the American Chemical Society 139(30):10561-10568, http://pubs.acs.org/doi/10.1021/jacs.7b05931

17. Delaney S, Barton JK (2003) Charge Transport in DNA Duplex/Quadruplex Conjugates. Biochemistry 42(48):14159-14165, https://doi.org/10.1021/bi0351965

18. Champdoré Md, Napoli LD, Montesarchio D, Piccialli G, Caminal C, Mulazzani QG, Navacchia ML, Chatgilialoglu C (2004) Excess elec- 
tron transfer in G-quadruplex. Chemical Communications (15):1756-1757, https://pubs.rsc.org/en/content/articlelanding/2004/cc/b404473h

19. Gervasio FL, Laio A, Iannuzzi M, Parrinello M (2004) Influence of DNA Structure on the Reactivity of the Guanine Radical Cation. Chemistry - A European Journal 10(19):4846-4852, http://doi.wiley.com/10.1002/chem.200400171

20. Martínez-Fernández L, Banyasz A, Markovitsi D, Improta R (2018) Topology Controls the Electronic Absorption and Delocalization of Electron Holes in Guanine Quadruplexes. Chemistry - A European Journal 24(57):15185-15189, https://onlinelibrary.wiley.com/doi/abs/10.1002/chem.201803222

21. Marcus RA, Sutin N (1985) Electron transfers in chemistry and biology. Biochimica et Biophysica Acta (BBA)-Reviews on Bioenergetics 811(3):265-322

22. Lech CJ, Phan AT, Michel-Beyerle ME, Voityuk AA (2013) Electron-Hole Transfer in G-Quadruplexes with Different Tetrad Stacking Geometries: A Combined QM and MD Study. The Journal of Physical Chemistry B 117(34):9851-9856, https://doi.org/10.1021/jp404788t

23. Woiczikowski PB, Kubař T, Gutiérrez R, Cuniberti G, Elstner M (2010) Structural stability versus conformational sampling in biomolecular systems: Why is the charge transfer efficiency in G4-DNA better than in double-stranded DNA? The Journal of Chemical Physics 133(3):035103, https://aip.scitation.org/doi/abs/10.1063/1.3460132

24. Lim KW, Amrane S, Bouaziz S, Xu W, Mu Y, Patel DJ, Luu KN, Phan AT (2009) Structure of the Human Telomere in K+ Solution: A Stable Basket-Type G-Quadruplex with Only Two G-Tetrad Layers. Journal of the American Chemical Society 131(12):4301-4309

25. Wang Y, Patel DJ (1993) Solution structure of the human telomeric repeat d[AG3(T2AG3)3] G-tetraplex. Structure 1(4):263-282, https://linkinghub.elsevier.com/retrieve/pii/0969212693900159

26. Ambrus A, Chen D, Dai J, Jones RA, Yang D (2005) Solution Structure of the Biologically Relevant G-Quadruplex Element in the Human c-MYC Promoter. Implications for G-Quadruplex Stabilization. Biochemistry 44(6):2048-2058, https://doi.org/10.1021/bi048242p

27. Luu KN, Phan AT, Kuryavyi V, Lacroix L, Patel DJ (2006) Structure of the Human Telomere in K+ Solution: An Intramolecular $(3+$ 1) G-Quadruplex Scaffold. Journal of the American Chemical Society 128(30):9963-9970, https://doi.org/10.1021/ja062791w

28. Winnerdy FR, Bakalar B, Maity A, Vandana JJ, Mechulam Y, Schmitt E, Phan AT (2019) NMR solution and X-ray crystal structures of a DNA molecule containing both right- and left-handed parallelstranded G-quadruplexes. Nucleic Acids Research 47(15):8272-8281, https://doi.org/10.1093/nar/gkz349

29. Bakalar B, Heddi B, Schmitt E, Mechulam Y, Phan AT (2019) A Minimal Sequence for Left-Handed G-Quadruplex Formation. Angewandte Chemie International Edition 58(8):2331-2335, 
https://onlinelibrary.wiley.com/doi/abs/10.1002/anie.201812628

30. Wu Q, Van Voorhis T (2005) Direct optimization method to study constrained systems within density-functional theory. Physical Review A 72(2), http://link.aps.org/doi/10.1103/PhysRevA.72.024502

31. $\mathrm{Wu}$ Q, Van Voorhis $\mathrm{T}$ (2006) Direct Calculation of Electron Transfer Parameters through Constrained Density Functional Theory. The Journal of Physical Chemistry A 110(29):9212-9218, http://dx.doi.org/10.1021/jp061848y

32. Salahub DR, Noskov SY, Lev B, Zhang R, Ngo V, Goursot A, Calaminici P, Köster AM, Alvarez-Ibarra A, Mejía-Rodríguez D, Řezáč J, Cailliez F, De la Lande A (2015) QM/MM Calculations with deMon2k. Molecules 20(3):4780-4812, https://www.mdpi.com/1420-3049/20/3/4780

33. Humphrey W, Dalke A, Schulten K (1996) VMD - Visual Molecular Dynamics. Journal of Molecular Graphics 14:33-38

34. Case DA, Belfon K, Ben-Shalom IY, Brozell SR, Cerutti DS, Cheatham TE, Cruzeiro VWD, Darden TA, Duke RE, Giambasu G, Gilson MK, Gohlke H, Goetz AW, Harris R, Izadi S, Ismailov SA, Kasavajhala K, Kovalenko A, Krasny R, Kurtzman T, Lee T, LeGrand S, Li P, Lin C, Liu J, Luchko T, Luo R, Man V, Merz KM, Miao Y, Mikhailovskii O, Monard G, Nguyen H, Onufriev A, Pan F, Pantano S, Qi R, Roe DR, Roitberg A, Sagui C, Schott-Verdugo S, Shen J, Simmerling C, Skrynnikov NR, Smith J, Swails JM, Walker RC, Wang J, Wilson L, Wolf RM, Wu X, Xiong Y, Xue Y, York DM, Kollman PA (2020) AMBER 2020. University of California, San Francisco

35. Ivani I, Dans PD, Noy A, Pérez A, Faustino I, Hospital A, Walther J, Andrio P, Goñi R, Balaceanu A, Portella G, Battistini F, Gelpí JL, González C, Vendruscolo M, Laughton CA, Harris SA, Case DA, Orozco M (2016) Parmbsc1: a refined force field for DNA simulations. Nature Methods 13(1):55-58

36. Jorgensen WL, Chandrasekhar J, Madura JD, Impey RW, Klein ML (1983) Comparison of simple potential functions for simulating liquid water. The Journal of chemical physics 79(2):926-935

37. Essmann U, Perera L, Berkowitz ML, Darden T, Lee H, Pedersen LG (1995) A smooth particle mesh Ewald method. The Journal of chemical physics 103(19):8577-8593

38. Roe DR, Cheatham TE (2013) Ptraj and cpptraj: Software for processing and analysis of molecular dynamics trajectory data. Journal of Chemical Theory and Computation 9(7):3084-3095

39. Köster AM, Calaminici P, Casida ME, Flores-Moreno R, Greudtner G, Goursot A, Heine T, Ipatov A, Janetzko F, del Campo JM, Patchkovskii S, Reveles JU, Vela A, Zuniga B, Salahub D (2006) demon2K. deMon developers, Cinvestav, Mexico City

40. de la Lande A, Salahub DR (2010) Derivation of interpretative models for long range electron transfer from constrained density functional theory. Journal of Molecular Structure: THEOCHEM 943(1):115-120, https://www.sciencedirect.com/science/article/pii/S0166128009007465 
41. Adamo C, Barone V (1999) Toward reliable density functional methods without adjustable parameters: The PBE0 model. The Journal of Chemical Physics 110(13):6158-6170, http://scitation.aip.org/content/aip/journal/jcp/110/13/10.1063/1.478522

42. Kendall RA, Dunning Jr TH, Harrison RJ (1992) Electron affinities of the first-row atoms revisited. Systematic basis sets and wave functions. The Journal of Chemical Physics 96(9):6796-6806, http://scitation.aip.org/content/aip/journal/jcp/96/9/10.1063/1.462569

43. Grimme S (2006) Semiempirical GGA-type density functional constructed with a long-range dispersion correction. Journal of Computational Chemistry 27(15):1787-1799, http://doi.wiley.com/10.1002/jcc.20495

44. Gillet N, Berstis L, Wu X, Gajdos F, Heck A, de la Lande A, Blumberger J, Elstner M (2016) Electronic Coupling Calculations for BridgeMediated Charge Transfer Using Constrained Density Functional Theory (CDFT) and Effective Hamiltonian Approaches at the Density Functional Theory (DFT) and Fragment-Orbital Density Functional Tight Binding (FODFTB) Level. Journal of Chemical Theory and Computation 12(10):4793-4805, https://doi.org/10.1021/acs.jctc.6b00564

45. Calaminici P, Janetzko F, Köster AM, Mejia-Olvera R, ZunigaGutierrez B (2007) Density functional theory optimized basis sets for gradient corrected functionals: 3d transition metal systems. The Journal of Chemical Physics 126(4):044108-10, http://scitation.aip.org/content/aip/journal/jcp/126/4/10.1063/1.2431643

46. Bultinck P, Ayers PW, Fias S, Tiels K, Van Alsenoy C (2007) Uniqueness and basis set dependence of iterative Hirshfeld charges. Chemical Physics Letters 444(1-3):205-208, http://linkinghub.elsevier.com/retrieve/pii/S000926140700930X

47. Wu Q, Van Voorhis T (2006) Extracting electron transfer coupling elements from constrained density functional theory. The Journal of Chemical Physics 125(16):164105, https://doi.org/10.1063/1.2360263, https://doi.org/10.1063/1.2360263

48. Blumberger J, Lamoureux G (2008) Reorganization free energies and quantum corrections for a model electron selfexchange reaction: comparison of polarizable and non-polarizable solvent models. Molecular Physics 106(12-13):1597-1611, https://www.tandfonline.com/doi/abs/10.1080/00268970802220112

49. de la Lande A, Alvarez-Ibarra A, Hasnaoui K, Cailliez F, Wu X, Mineva T, Cuny J, Calaminici P, López-Sosa L, Geudtner G, Navizet I, Garcia Iriepa C, Salahub DR, Köster AM (2019) Molecular simulations with in-demon2k qm/mm, a tutorial-review. Molecules 24(9), https://www.mdpi.com/1420$3049 / 24 / 9 / 1653$ 